\title{
QSPR Analysis of Chemical Graph Theory
}

\author{
Rachanna Kanabur $^{\mathrm{a}, *}$, S.K.Giregol ${ }^{\mathrm{a}}$, Akshata Nagaral, Priyanka Badave, Rekha Sannakkai, Renuka Sasalatti ${ }^{\mathrm{a}}$ \\ ${ }^{a}$ Department of Mathematics BLDEA's, Commerce, B.H.S. Arts and T.G.P. Science College, Jamkhandi Karnataka, India.
}

\begin{abstract}
In this paper, we find the values of four important degree-based topological indices of molecular graph of alkane isomers. Further, we show that these parameters are highly correlated with physical properties of alkane isomers. . C2019 All rights reserved.
\end{abstract}

Keywords: Alkane isomers,, Degree-based topological indices.

2010 MSC: 05C10, 57M15, 57M25.

\section{Introduction}

Let $G=(V, E)$ be a graph with $n$ vertices and $m$ edges. The degree of a vertex $u \in V(G)$ is denoted by $d_{G}(u)$ and is the number of vertices that are adjacent to $u$. The edge connecting the vertices $u$ and $v$ is denoted by uv [4].

Chemical graph theory is the branch of mathematical chemistry. It is concerned with handling chemical graphs that represent chemical system. Hence chemical graph theory deals with analysis of all consequences of connectivity in a chemical system. It has found to be a useful tool in QSAR (Quantitative Structure-Activity Relationship) and QSPR (Quantitative Structure-Property Relationship) [8, 5, 14]. Numerous studies have been made relating to the above mentioned fields by using what are called topological indices. In 1975, Randic [10] proposed a topological index that has become one of the most widely used in both QSAR and QSPR studies.

Mathematical chemistry is a branch of theoretical chemistry for discussion and prediction of the molecular structure using mathematical methods without necessarily referring to quantum mechanics. Chemical graph theory is a branch of mathematical chemistry which applies graph theory to mathematical modelling of chemical phenomena $[4,2,11]$. This theory had an important effect on the development of the chemical sciences.

\footnotetext{
${ }^{*}$ Corresponding author

Email addresses: rachukanabur@gmail.com (Rachanna Kanabur), oladalalaa@yahoo.com (S.K.Giregol), olimat_anas@yahoo.com (Akshata Nagaral, Priyanka Badave, Rekha Sannakkai, Renuka Sasalatti)

doi:10.31559/glm2020.9.1.2
} 


\section{Computing Some New Degree-Based Topological Indices}

We consider the following most studied degree based topological indices for QSPR study [12, 14, 15, 16].

Definition 2.1. Let $G=(V, E)$ be a molecular graph, and $d_{G}(u)$ is the degree of the vertex $u$, then Arithmetic-Geometric Index $\left(A G_{1}\right.$ index $)$ of $G$ is defined as

$$
A G_{1}(G)=\sum_{u, v \in E(G)} \frac{d_{G}(u)+d_{G}(v)}{2 \sqrt{d_{G}(u) \cdot d_{G}(v)}}
$$

Where, $\left(A G_{1}\right.$ index $)$ is considered for distinct vertices.

The above equation is the sum of the ratio of the Arithmetic mean and Geometric mean of $u$ and $v$, where $d_{G}(u)$ (or $\left.d_{G}(v)\right)$ denotes the degree of the vertex $u($ or $v)$.

Definition 2.2. The $S K$ index of a graph $G=(V, E)$ is defined as $S K(G)=\sum_{u, v \in E(G)} \frac{d_{G}(u)+d_{G}(v)}{2}$, where $\mathrm{d}_{\mathrm{G}}(\mathrm{u})$ and $\mathrm{d}_{\mathrm{G}}(v)$ are the degrees of the vertices $u$ and $v$ in $\mathrm{G}$.

Definition 2.3. The $S K_{1}$ index of a graph $G=(V, E)$ is defined as $S K_{1}(G)=\sum \cdot u_{u, v \in E(G)} \frac{d_{G}(u) \cdot d_{G}(v)}{2}$, where $d_{G}(u)$ and $d_{G}(v)$ are the degrees of the vertices $u$ and $v$ in $G$.

Definition 2.4. The $S K_{2}$ index of a graph $G=(V, E)$ is defined as $S K_{2}(G)=\sum_{u, v \in E(G)}\left(\frac{d_{G}(u)+d_{G}(v)}{2}\right)^{2}$, where $d_{G}(u)$ and $d_{G}(v)$ are the degrees of the vertices $u$ and $v$ in $G$.

\section{Motivation}

By looking at the earlier results QSPR Analysis of Certain Degree-Based Topological Indices and QSPR Analysis of Degree-Based Topological Indices with physical properties of Benzenoid Hydrocarbons $[7,5,6,13]$.

\section{Data Sources and Analytical Method}

Energy of Alkane isomers are taken from [19, 7, 12, 5]. Seven physicochemical properties of Alkane isomers have been selected on the availability of a suitable body of data: boiling point (BP), critical temperature (CT), critical pressure (CP), Molar volume (CV), Molar refraction (MR), Heat of Vaporization (HV), Surface tension (ST). The values are compiled in Table 2.

\section{Results and Discussion}

In Table 3, the correlation coefficient of topological indices with physicochemical properties of alkane isomers are found to be good.

\subsection{Regression Models}

We have tested the following linear regression model

$$
\mathrm{P}=\mathrm{a}+\mathrm{b}(\mathrm{TI})
$$

where, $\mathrm{P}=$ Physical property, $\mathrm{TI}=$ Topological Index. Using (4.1), we have obtained the following different linear models for each degree-based topological index, which are listed below. 
Table 1: The topological indices (AG1 Index, SK Index, SK1 Index and SK2 Index) values of Alkane Isomers.

\begin{tabular}{|c|c|c|c|c|c|}
\hline S.NO. & Compound Name & $\begin{array}{c}\text { AG1 } \\
\text { Index }\end{array}$ & $\begin{array}{c}\text { SK } \\
\text { Index }\end{array}$ & $\begin{array}{c}\text { SK1 } \\
\text { Index }\end{array}$ & $\begin{array}{c}\text { SK2 } \\
\text { Index }\end{array}$ \\
\hline 1 & Butane & 3.1213 & 5 & 4 & 8.5 \\
\hline 2 & 2-methyl propane & 3.46410 & 6 & 4.5 & 12 \\
\hline 3 & Pentane & 4.123 & 7 & 6 & 12.5 \\
\hline 4 & 2-methyl butane & 4.3906 & 8 & 7 & 16.5 \\
\hline 5 & 2,2 dimethyl propane & 5 & 10 & 8 & 25 \\
\hline 6 & Hexane & 5.1213 & 9 & 8 & 16.5 \\
\hline 7 & 2-methyl pentane & 5.2359 & 10 & 9 & 20.5 \\
\hline 8 & 3- methyl pentane & 5.3172 & 10 & 9.5 & 21 \\
\hline 9 & 2,2-dimethyl butane & 5.8713 & 12 & 11 & 30 \\
\hline 10 & 2,3- dimethyl butane & 5.6188 & 11 & 10.5 & 25 \\
\hline 11 & Heptanes & 6.1213 & 11 & 10 & 20.5 \\
\hline 12 & 2-methyl hexane & 6.3906 & 12 & 11 & 24.5 \\
\hline 13 & 3- methyl hexane & 6.5767 & 12 & 11.5 & 25 \\
\hline 14 & 3-ethylpentane & 6.2438 & 12 & 12 & 25.5 \\
\hline 15 & 2,2-dimethyl pentane & 6.8713 & 14 & 13 & 34 \\
\hline 16 & 2,3-dimethyl pentane & 6.54530 & 13 & 13 & 29.5 \\
\hline 17 & 2,4-dimethyl pentane & 6.6600 & 13 & 12 & 28.5 \\
\hline 18 & 3,3-dimethyl pentane & 6.7426 & 14 & 14 & 35 \\
\hline 19 & Octane & 7.1213 & 13 & 12 & 24.5 \\
\hline 20 & 2-methylheptane & 7.3906 & 14 & 13 & 28.5 \\
\hline 21 & 3- methylheptane & 7.3172 & 14 & 13.5 & 29 \\
\hline 22 & 4- methylheptane & 7.3172 & 14 & 13.5 & 29 \\
\hline 23 & 3-ethylhexane & 7.2438 & 14 & 14 & 29.5 \\
\hline 24 & 2,2-dimethylhexane & 7.8713 & 16 & 15 & 38 \\
\hline 25 & 2,3-dimethylhexane & 7.5453 & 15 & 15 & 33.5 \\
\hline 26 & 2,4-dimethylhexane & 7.5866 & 15 & 14.5 & 33 \\
\hline 27 & 2,5- dimethylhexane & 7.6600 & 15 & 14 & 32.5 \\
\hline 28 & 3,3-dimethylhexane & 7.7426 & 16 & 16 & 39 \\
\hline 29 & 3,4-dimethylhexane & 7.4719 & 15 & 15.5 & 34 \\
\hline 30 & 3-ethyl-2-methylpentane & 7.4719 & 15 & 15.5 & 34 \\
\hline 31 & 3-ethyl-3-methylpentane & 7.6139 & 16 & 17 & 40 \\
\hline 32 & $2,2,3$-trimethylpentane & 7.9963 & 17 & 17.5 & 43.5 \\
\hline 33 & 2,2,4-trimethylpentane & 8.1406 & 17 & 16 & 42 \\
\hline 34 & 2,3,3- trimethylpentane & 7.9410 & 17 & 18 & 44 \\
\hline 35 & $2,3,4$ - trimethylpentane & 8.4401 & 16 & 16.5 & 38 \\
\hline 36 & Nonane & 8.1213 & 15 & 14 & 28.5 \\
\hline 37 & 2-methyloctane & 8.3906 & 16 & 15 & 32.5 \\
\hline 38 & 3-methyloctane & 8.3172 & 16 & 15.5 & 33 \\
\hline 39 & 4-methyloctane & 8.3172 & 16 & 15.5 & 33 \\
\hline 40 & 3-ethylheptane & 8.2438 & 16 & 16 & 33.5 \\
\hline 41 & 4- ethylheptane & 8.2438 & 16 & 16 & 33.5 \\
\hline 42 & 2,2-dimethylheptane & 8.8713 & 18 & 17 & 42 \\
\hline 43 & 2,3- dimethylheptane & 8.5453 & 17 & 17 & 37.5 \\
\hline 44 & 2,4- dimethylheptane & 10.8960 & 17 & 16.5 & 37 \\
\hline 45 & 2,5-dimethylheptane & 8.5866 & 17 & 16.5 & 37 \\
\hline 46 & 2,6- dimethylheptane & 8.6600 & 17 & 16 & 36.5 \\
\hline 47 & 3,3-dimethylheptane & 8.7426 & 18 & 18 & 43 \\
\hline 48 & 3,4- dimethylheptane & 7.4719 & 17 & 17.5 & 38 \\
\hline 49 & 3,5- dimethylheptane & 8.5132 & 17 & 17 & 37.5 \\
\hline 50 & 4,4- dimethylheptane & 8.7426 & 18 & 18 & 43 \\
\hline 51 & 3-ethyl-2-methylhexane & 8.4719 & 17 & 17.5 & 38 \\
\hline 52 & 4-ethyl-2-methylhexane & 8.5132 & 17 & 17 & 37.5 \\
\hline 53 & 3-ethyl-3-methylhexane & 7.0229 & 18 & 19 & 44 \\
\hline 54 & 2,2,4-trimethylhexane & 9.0672 & 19 & 18.5 & 46.5 \\
\hline 55 & 2,2,5-trimethylhexane & 9.1406 & 19 & 18 & 46 \\
\hline 56 & 2,3,3-trimethylhexane & 8.9410 & 19 & 20 & 48 \\
\hline 57 & 2,3,4- trimethylhexane & 8.7000 & 18 & 19 & 42.6 \\
\hline 58 & $2,3,5$ - trimethylhexane & 8.8147 & 18 & 18 & 41.5 \\
\hline 59 & 3,3,4- trimethylhexane & 8.8676 & 19 & 20.5 & 48.5 \\
\hline 60 & 3,3-diethylpentane & 8.4852 & 18 & 20 & 40.5 \\
\hline 61 & 2,2-dimethyl-3-ethylpentane & 8.9229 & 19 & 20 & 44.75 \\
\hline 62 & 2,3-dimethyl-3ethylpentane & 8.8124 & 19 & 21 & 49 \\
\hline 63 & 2,4-dimethyl-3-ethylpentane & 8.7000 & 18 & 19 & 42.5 \\
\hline 64 & 2,2,3,3-tetramethylpentane & 9.3713 & 21 & 23 & 58.5 \\
\hline 65 & $2,2,3,4$ - tetramethylpentane & 9.2244 & 20 & 21 & 52 \\
\hline 66 & $2,2,4,4$ - tetramethylpentane & 9.6213 & 21 & 20 & 55.5 \\
\hline 67 & 2,3,3,4- tetramethylpentane & 9.1395 & 20 & 22 & 53 \\
\hline
\end{tabular}

1. $A G_{1} \operatorname{Index}\left(A G_{1}(G)\right)$

$$
\begin{aligned}
\mathrm{Bp} & =3.992+0.033 \mathrm{AG}_{1}(\mathrm{G}) \\
\mathrm{CT} & =-0.976+0.03 \mathrm{AG}_{1}(\mathrm{G}) \\
\mathrm{CP} & =18.622-0.414 \mathrm{AG}_{1}(\mathrm{G}) \\
\mathrm{MV} & =-3.525+0.069 \mathrm{AG}_{1}(\mathrm{G}) \\
\mathrm{ST} & =-2.608+0.495 \mathrm{AG}_{1}(\mathrm{G})
\end{aligned}
$$


Table 2: The physical and chemical properties (BP, MV, MR, HV, CT, CP, ST) of Alkane Isomers. \begin{tabular}{|l|c|c|c|c|c|c|c|c|}
\hline S.NO. & Compound Name & Bp(0C) & $\mathrm{mv}(\mathrm{cm} 3)$ & $\mathrm{mr}(\mathrm{cm} 3)$ & $\mathrm{hv}(\mathrm{kJ})$ & $\mathrm{ct}(0 \mathrm{C})$ & $\mathrm{cp}(\mathrm{atm})$ & St(dyne/cm) \\
\hline
\end{tabular}

\begin{tabular}{|c|c|c|c|c|c|c|c|c|}
\hline 1 & Butane & -0.500 & & 20.68 & 22.44 & 152.01 & 37.47 & 12.46 \\
\hline 2 & 2-methyl propane & -11.730 & & 20.85 & 21.6 & 134.98 & 36 & 14.1 \\
\hline 3 & Pentane & 36.074 & 115.205 & 25.2656 & 26.42 & 196.62 & 33.31 & 16.00 \\
\hline 4 & 2-methyl butane & 27.852 & 116.426 & 25.2923 & 24.59 & 187.70 & 32.9 & 15.00 \\
\hline 5 & 2,2 dimethyl propane & 9.503 & 112.074 & 25.7243 & 21.78 & 160.60 & 31.57 & 12.05 \\
\hline 6 & Hexane & 68.740 & 130.688 & 29.9066 & 31.55 & 234.70 & 29.92 & 18.42 \\
\hline 7 & 2-methyl pentane & 60.271 & 131.933 & 29.9459 & 29.86 & 224.90 & 29.95 & 17.38 \\
\hline 8 & 3- methyl pentane & 63.282 & 129.717 & 29.8016 & 30.27 & 231.20 & 30.83 & 18.12 \\
\hline 9 & 2,2-dimethyl butane & 49.741 & 132.744 & 29.9347 & 27.69 & 216.20 & 30.67 & 16.30 \\
\hline 10 & 2,3- dimethyl butane & 57.988 & 130.240 & 29.8104 & 29.12 & 227.10 & 30.99 & 17.37 \\
\hline 11 & Heptanes & 98.427 & 146.540 & 34.5504 & 36.55 & 267.55 & 27.01 & 20.26 \\
\hline 12 & 2-methyl hexane & 90.052 & 147.656 & 34.5908 & 34.80 & 257.90 & 27.2 & 19.29 \\
\hline 13 & 3- methyl hexane & 91.850 & 145.821 & 34.4597 & 35.08 & 262.40 & 28.1 & 19.79 \\
\hline 14 & 3-ethylpentane & 93.475 & 143.517 & 34.2827 & 35.22 & 267.60 & 28.6 & 20.44 \\
\hline 15 & 2,2-dimethyl pentane & 79.197 & 148.695 & 34.6166 & 32.43 & 247.70 & 28.4 & 18.02 \\
\hline 16 & 2,3-dimethyl pentane & 89.784 & 144.153 & 34.3237 & 34.24 & 264.60 & 29.2 & 19.96 \\
\hline 17 & 2,4- dimethyl pentane & 80.500 & 148.949 & 34.6192 & 32.88 & 247.10 & 27.4 & 18.15 \\
\hline 18 & 3,3- dimethyl pentane & 86.064 & 144.530 & 34.3323 & 33.02 & 263.00 & 30 & 19.59 \\
\hline 19 & Octane & 125.665 & 162.592 & 39.1922 & 41.48 & 296.20 & 24.64 & 21.76 \\
\hline 20 & 2-methylheptane & 117.647 & 163.663 & 39.2316 & 39.68 & 288.00 & 24.8 & 20.60 \\
\hline 21 & 3- methylheptane & 118.925 & 161.832 & 39.1001 & 39.83 & 292.00 & 25.6 & 21.17 \\
\hline 22 & 4- methylheptane & 117.709 & 162.105 & 39.1174 & 39.67 & 290.00 & 25.6 & 21.00 \\
\hline 23 & 3-ethylhexane & 118.53 & 160.07 & 38.94 & 39.40 & 292.00 & 25.74 & 21.51 \\
\hline 24 & 2,2-dimethylhexane & 10.84 & 164.28 & 39.25 & 37.29 & 279.00 & 25.6 & 19.60 \\
\hline 25 & 2,3-dimethylhexane & 115.607 & 160.39 & 38.98 & 38.79 & 293.00 & 26.6 & 20.99 \\
\hline 26 & 2,4- dimethylhexane & 109.42 & 163.09 & 39.13 & 37.76 & 282.00 & 25.8 & 20.05 \\
\hline 27 & 2,5-dimethylhexane & 109.10 & 164.69 & 39.25 & 37.86 & 279.00 & 25 & 19.73 \\
\hline 28 & 3,3- dimethylhexane & 111.96 & 160.87 & 39.00 & 37.93 & 290.84 & 27.2 & 20.63 \\
\hline 29 & 3,4- dimethylhexane & 117.72 & 158.81 & 38.84 & 39.02 & 298.00 & 27.4 & 21.64 \\
\hline 30 & 3-ethyl-2-methylpentane & 115.65 & 158.79 & 38.83 & 38.52 & 295.00 & 27.4 & 21.52 \\
\hline 31 & 3-ethyl-3-methylpentane & 118.25 & 157.02 & 38.71 & 37.99 & 305.00 & 28.9 & 21.99 \\
\hline 32 & 2,2,3-trimethylpentane & 109.84 & 159.52 & 38.92 & 36.91 & 294.00 & 28.2 & 20.67 \\
\hline 33 & 2,2,4- trimethylpentane & 99.23 & 165.08 & 39.26 & 35.13 & 271.15 & 25.5 & 18.77 \\
\hline 34 & 2,3,3- trimethylpentane & 114.76 & 157.29 & 38.76 & 37.22 & 303.00 & 29 & 21.56 \\
\hline 35 & 2,3,4-trimethylpentane & 113.46 & 158.85 & 38.86 & 37.61 & 295.00 & 27.6 & 21.14 \\
\hline 36 & Nonane & 150.79 & 178.71 & 43.84 & 46.44 & 322.00 & 22.74 & 22.92 \\
\hline 37 & 2-methyloctane & 143.26 & 179.77 & 43.87 & 44.65 & 315.00 & 23.6 & 21.88 \\
\hline 38 & 3-methyloctane & 144.18 & 177.95 & 43.72 & 44.75 & 318.00 & 23.7 & 22.34 \\
\hline 39 & 4-methyloctane & 142.48 & 178.15 & 43.76 & 44.75 & 318.30 & 23.06 & 22.34 \\
\hline 40 & 3-ethylheptane & 143.00 & 176.41 & 43.64 & 44.81 & 318.00 & 23.98 & 22.81 \\
\hline 41 & 4- ethylheptane & 141.20 & 175.68 & 43.49 & 44.81 & 318.30 & 23.98 & 22.81 \\
\hline 42 & 2,2-dimethylheptane & 132.69 & 180.50 & 43.91 & 42.28 & 302.00 & 22.8 & 20.80 \\
\hline 43 & 2,3- dimethylheptane & 140.50 & 176.65 & 43.63 & 43.79 & 315.00 & 23.79 & 22.34 \\
\hline 44 & 2,4-dimethylheptane & 133.50 & 179.12 & 43.73 & 42.87 & 306.00 & 22.7 & 23.30 \\
\hline 45 & 2,5- dimethylheptane & 136.00 & 179.37 & 43.84 & 43.87 & 307.80 & 22.7 & 21.30 \\
\hline 46 & 2,6- dimethylheptane & 135.21 & 180.91 & 43.92 & 42.82 & 306.00 & 23.7 & 20.83 \\
\hline 47 & 3,3-dimethylheptane & 137.00 & 176.897 & 43.6870 & 42.66 & 314.00 & 24.19 & 22.01 \\
\hline 48 & 3,4-dimethylheptane & 140.600 & 175.349 & 43.5473 & 43.84 & 322.70 & 24.77 & 22.80 \\
\hline 49 & 3,5-dimethylheptane & 136.000 & 177.386 & 43.6379 & 42.98 & 312.30 & 23.59 & 21.77 \\
\hline 50 & 4,4-dimethylheptane & 135.200 & 176.897 & 43.6022 & 42.66 & 317.80 & 24.18 & 22.01 \\
\hline 51 & 3-ethyl-2-methylhexane & 138.000 & 175.445 & 43.6550 & 43.84 & 322.70 & 24.77 & 22.80 \\
\hline 52 & 4-ethyl-2-methylhexane & 133.800 & 177.386 & 43.6472 & 42.98 & 330.30 & 25.56 & 21.77 \\
\hline 53 & 3-ethyl-3-methylhexane & 140.600 & 173.077 & 43.2680 & 44.04 & 327.20 & 25.66 & 23.22 \\
\hline 54 & 2,2,4-trimethylhexane & 126.540 & 179.220 & 43.7638 & 40.57 & 301.00 & 23.39 & 20.51 \\
\hline 55 & 2,2,5- trimethylhexane & 124.084 & 181.346 & 43.9356 & 40.17 & 296.60 & 22.41 & 20.04 \\
\hline 56 & 2,3,3-trimethylhexane & 137.680 & 173.780 & 43.4347 & 42.23 & 326.10 & 25.56 & 22.41 \\
\hline 57 & 2,3,4- trimethylhexane & 139.000 & 173.498 & 43.4917 & 42.93 & 324.20 & 25.46 & 22.80 \\
\hline 58 & 2,3,5- trimethylhexane & 131.340 & 177.656 & 43.6474 & 41.42 & 309.40 & 23.49 & 21.27 \\
\hline 59 & 3,3,4- trimethylhexane & 140.460 & 172.055 & 43.3407 & 42.28 & 330.60 & 26.45 & 23.27 \\
\hline 60 & 3,3-diethylpentane & 146.168 & 170.185 & 43.1134 & 43.36 & 342.80 & 26.94 & 23.75 \\
\hline 61 & 2,2-dimethyl-3-ethylpentane & 133.830 & 174.537 & 43.4571 & 42.02 & 322.60 & 25.96 & 22.38 \\
\hline 62 & 2,3-dimethyl-3-ethylpentane & 142.000 & 170.093 & 42.9542 & 42.55 & 338.60 & 26.94 & 23.87 \\
\hline 63 & 2,4-dimethyl-3-ethylpentane & 136.730 & 173.804 & 43.4037 & 42.93 & 324.20 & 25.46 & 22.80 \\
\hline 64 & 2,2,3,3-tetramethylpentane & 140.274 & 169.495 & 43.2147 & 41.00 & 334.50 & 27.04 & 23.38 \\
\hline 65 & 2,2,3,4- tetramethylpentane & 133.016 & 173.557 & 43.4359 & 41.00 & 319.60 & 25.66 & 21.98 \\
\hline 66 & $2,2,4,4$ - tetramethylpentane & 122.284 & 178.256 & 43.8747 & 38.10 & 301.60 & 24.58 & 20.37 \\
\hline 67 & 2,3,3,4- tetramethylpentane & 141.551 & 169.928 & 43.2016 & 41.75 & 334.50 & 26.85 & 23.31 \\
\hline
\end{tabular}

Table 3: Correlation of topological indices with physicochemical properties of Alkane isomers.

\begin{tabular}{llllllll}
\hline & $\mathrm{Bp}\left({ }^{0}\right)$ & $\mathrm{ct}\left({ }^{0} \mathrm{C}\right)$ & $\mathrm{cp}(\mathrm{atm})$ & $\mathrm{m} v\left(\mathrm{~cm}^{3}\right)$ & $\mathrm{st}\left(\frac{\mathrm{dyne}}{\mathrm{cm}}\right)$ & $\mathrm{hv}(\mathrm{kJ})$ & $\mathrm{mr}\left(\mathrm{cm}^{3}\right)$ \\
\hline $\mathrm{AG}_{1}$ & 0.8463 & 0.8946 & -0.8514 & 0.9186 & 0.8308 & 0.8614 & 0.9467 \\
$\mathrm{SK}$ & 0.8211 & 0.8945 & -0.7661 & 0.8736 & 0.8097 & 0.8176 & 0.927 \\
$\mathrm{SK}_{1}$ & 0.8112 & 0.8959 & -0.6730 & 0.8047 & 0.8290 & 0.7910 & 0.8864 \\
$\mathrm{SK}_{2}$ & 0.6841 & 0.7784 & -0.5897 & 0.8047 & 0.6870 & 0.6522 & 0.7987 \\
\hline
\end{tabular}




$$
\begin{gathered}
\mathrm{HV}=-0.7+0.216 \mathrm{AG}_{1}(\mathrm{G}) \\
\mathrm{MR}=-1.586+0.236 \mathrm{AG}_{1}(\mathrm{G})
\end{gathered}
$$

2. SK Index

$$
\begin{gathered}
\mathrm{Bp}=7.116+0.074 \mathrm{SK}(\mathrm{G}) \\
\mathrm{CT}=-4.689+0.069 \mathrm{SK}(\mathrm{G}) \\
\mathrm{CP}=38.327-0.867 \mathrm{SK}(\mathrm{G}) \\
\mathrm{MV}=-9.553+0.155 \mathrm{SK}(\mathrm{G}) \\
\mathrm{ST}=-7.885+1.122 \mathrm{SK}(\mathrm{G}) \\
\mathrm{HV}=-3.07+0.478 \mathrm{SK}(\mathrm{G}) \\
\mathrm{MR}=-5.664+0.538 \mathrm{SK}(\mathrm{G})
\end{gathered}
$$

3. $\mathrm{SK}_{1}$ Index

$$
\begin{gathered}
\mathrm{Bp}=5.565+0.087 \mathrm{SK}_{1}(\mathrm{G}) \\
\mathrm{CT}=-8.611+0.083 \mathrm{SK}_{1}(\mathrm{G}) \\
\mathrm{CP}=39.199-0.905 \mathrm{SK}_{1}(\mathrm{G}) \\
\mathrm{MV}=-12.542+0.172 \mathrm{SK}_{1}(\mathrm{G}) \\
\mathrm{ST}=-13.031+1.365 \mathrm{SK}_{1}(\mathrm{G}) \\
\mathrm{HV}=-5.945+0.549 \mathrm{SK}_{1}(\mathrm{G}) \\
\mathrm{MR}=-8.648+0.611 \mathrm{SK}_{1}(\mathrm{G})
\end{gathered}
$$

4. $\mathrm{SK}_{2}$ Index

$$
\begin{gathered}
\mathrm{Bp}=15.014+0.182 \mathrm{SK}_{2}(\mathrm{G}) \\
\mathrm{CT}=-16.102+0.178 \mathrm{SK}_{2}(\mathrm{G}) \\
\mathrm{CP}=87.24-1.965 \mathrm{SK}_{2}(\mathrm{G}) \\
\mathrm{MV}=-12.542+0.172 \mathrm{SK}_{2}(\mathrm{G}) \\
\mathrm{ST}=-22.813+2.802 \mathrm{SK}_{2}(\mathrm{G}) \\
\mathrm{HV}=-8.052+1.122 \mathrm{SK}_{2}(\mathrm{G}) \\
\mathrm{MR}=-18.07+1.364 \mathrm{SK}_{2}(\mathrm{G})
\end{gathered}
$$

By inspection of the data in Table 4 to Table 7 , it is possible to draw a number of conclusions for the given topological indices like invariants.

The QSPR study of $A G_{1}(G)$ index reveals that can be useful in predicting the boiling point, critical temperature, critical pressure, Molar volume, Surface tension, Heat of Vaporisation, Molar refraction of alkane isomers also from Table 4, one can easily verify that $A G_{1}(G)$ index shows good correlation with all physical properties of alkane isomers. We can see that the correlation coefficient value of $A G_{1}(G)$ index with physical properties of alkane isomers lies between -0.8514 to 0.9467 .

Further, the correlation coefficient value of $A G_{1}(G)$ index is very high for the MR of alkane isomers with $r=0.9467$. Thus the QSPR study reveals that the $A G_{1}(G)$ index is an useful tool in predicting the 
Table 4: Statistical parameters for the linear QSPR model for $A G_{1}(G)$.

\begin{tabular}{lllll}
\hline Physical Properties & $\mathrm{N}$ & $\mathrm{a}$ & $\mathrm{b}$ & $\mathrm{r}$ \\
\hline Boiling point & 67 & 3.992 & 0.033 & 0.8463 \\
Critical temperature & 67 & -0.976 & 0.03 & 0.8946 \\
Critical pressure & 67 & 18.622 & -0.414 & -0.8514 \\
Molar volume & 65 & -3.525 & 0.069 & 0.9186 \\
ST & 67 & -2.608 & 0.495 & 0.8308 \\
HV & 67 & -0.7 & 0.216 & 0.8614 \\
MR & 67 & -1.586 & 0.236 & 0.9467 \\
\hline
\end{tabular}

Table 5: Statistical parameters for the linear QSPR model for SK (G).

\begin{tabular}{lllll}
\hline Physical Properties & $\mathrm{N}$ & $\mathrm{a}$ & $\mathrm{b}$ & $\mathrm{r}$ \\
\hline Boiling point & 67 & 7.116 & 0.074 & 0.8211 \\
Critical temperature & 67 & -4.689 & 0.069 & 0.8945 \\
Critical pressure & 67 & 38.327 & -0.867 & -0.7661 \\
Molar volume & 65 & -9.553 & 0.155 & 0.8736 \\
ST & 67 & -7.885 & 1.122 & 0.8097 \\
HV & 67 & -3.07 & 0.478 & 0.8176 \\
MR & 67 & -5.664 & 0.538 & 0.927 \\
\hline
\end{tabular}

Table 6: Statistical parameters for the linear QSPR model for $\mathrm{SK}_{1}(\mathrm{G})$.

\begin{tabular}{lllll}
\hline Physical Properties & $\mathrm{N}$ & $\mathrm{a}$ & $\mathrm{b}$ & $\mathrm{r}$ \\
\hline Boiling point & 67 & 5.565 & 0.087 & 0.8112 \\
Critical temperature & 67 & -8.611 & 0.083 & 0.8959 \\
Critical pressure & 67 & 39.199 & -0.905 & -0.673 \\
Molar volume & 65 & -12.542 & 0.172 & 0.8047 \\
ST & 67 & -13.031 & 1.365 & 0.829 \\
HV & 67 & -5.945 & 0.549 & 0.791 \\
MR & 67 & -8.648 & 0.611 & 0.8864 \\
\hline
\end{tabular}

Table 7: Statistical parameters for the linear QSPR model for $\mathrm{SK}_{2}(\mathrm{G})$.

\begin{tabular}{lllll}
\hline Physical Properties & $\mathrm{N}$ & $\mathrm{a}$ & $\mathrm{b}$ & $\mathrm{r}$ \\
\hline Boiling point & 67 & 15.014 & 0.182 & 0.6841 \\
Critical temperature & 67 & -16.102 & 0.178 & 0.7784 \\
Critical pressure & 67 & 87.24 & -1.965 & -0.673 \\
Molar volume & 65 & -12.542 & 0.172 & 0.8047 \\
ST & 67 & -22.813 & 2.802 & 0.687 \\
HV & 67 & -8.052 & 1.122 & 0.6522 \\
MR & 67 & -18.07 & 1.364 & 0.7987 \\
\hline
\end{tabular}

physical properties of alkane isomers. The QSPR study of SK (G) index reveals that can be useful in predicting the boiling point, critical temperature, critical pressure, Molar volume, Surface tension, Heat of Vaporisation, Molar refraction of alkane isomers also from Table 5, one can easily verify that SK (G) index index shows good correlation with all physical properties of alkane isomers. We can see that the corre- 
lation coefficient value of SK (G) index with physical properties of alkane isomers lies between -0.7661 to 0.927. Further, the correlation coefficient value of SK $(G)$ index index is very high for the MR of alkane isomers with $r=0.927$. Thus the QSPR study reveals that the $S K(G)$ index index is an useful tool in predicting the physical properties of alkane isomers.

The QSPR study of $\mathrm{SK}_{1}(\mathrm{G})$ index reveals that can be useful in predicting the boiling point, critical temperature, critical pressure, Molar volume, Surface tension, Heat of Vaporisation, Molar refraction of alkane isomers also from Table 6, one can easily verify that $\mathrm{SK}_{1}(\mathrm{G})$ index shows good correlation with all physical properties of alkane isomers. We can see that the correlation coefficient value of $S K_{1}(G)$ index with physical properties of alkane isomers lies between -0.673 to 0.8959 . Further, the correlation coefficient value of $S_{K_{1}}$ index is very high for the CT of alkane isomers with $r=0.8959$. Thus the QSPR study reveals that the $\mathrm{SK}_{1}(\mathrm{G})$ index is an useful tool in predicting the physical properties of alkane isomers.

The QSPR study of $\mathrm{SK}_{2}(\mathrm{G})$ index reveals that can be useful in predicting the boiling point, critical temperature, critical pressure, Molar volume, Surface tension, Heat of Vaporisation, Molar refraction of alkane isomers also from Table 7, one can easily verify that $\mathrm{SK}_{2}(\mathrm{G})$ index index shows good correlation with all physical properties of alkane isomers. We can see that the correlation coefficient value of $\mathrm{SK}_{2}(\mathrm{G})$ index with physical properties of alkane isomers lies between -0.673 to 0.7987 . Further, the correlation coefficient value of $\mathrm{SK}_{2}(\mathrm{G})$ index is very high for the $\mathrm{MR}$ of alkane isomers with $\mathrm{r}=0.7987$. Thus the QSPR study reveals that the $\mathrm{SK}_{2}(\mathrm{G})$ index is an useful tool in predicting the physical properties of alkane isomers.

\section{References}

[1] J. Devillers, A. T. Balban, Topological indices and related descriptors in QSAR and QSPR, Gordon and Breach Science Publishers, Amsterdam, Netherland, (1999).

[2] M.V.Diudea, I. Gutman, J.Lorentz, Molecular Topology, Babes-Bolyai University, Romania, (2001).

[3] I. Gutman, Degree-based topological indices, Croat. Chem. Acta, 86(2013), 251-361.

[4] F. Harary, Graph theory, Addison-Wesely, Reading mass (1969).

[5] S.M. Hosamani, Deepa M. Perigidad, Shruti Y. Jamagoud Yallavva B. Maled and SharadaGavade , QSPR Analysis of Certain Degree Based Topological Indices, Journal of Statistics Applications and Probability, 2016. https:/ / doi.org/10.18576/jsap/060211

[6] S.M.Hosamani, Correlation of Domination Parameters with physico-chemical properties of Octane isomers, Applied Mathematics and Nonlinear Sciences, 1 (2) (2016), 345-352.

[7] R. Kanabur, V.S. Shigehalli, , QSPR Analysis of Degree-Based Topological Indices with physical properties of Benzenoid Hydrocarbons, General Letters in Mathematics, 2 (3), (2017), 150-169. https:/ / doi.org/10.31559/glm2016.2.3.6

[8] Minati Kuanar, Saroj K Kuanar, Bijay K Mishra, Correlation of line graph parameters with physical properties of octane isomers, Indian Journal of Chemistry, 38A (1999), 525-528.

[9] Mustafa ÖZKAN, Figen ÖKE, Repeat codes, Even codes, Odd codes and Their equivalence, General Letters in Mathematics, 2 (1), 110-118. doi:10.31559/glm2016.2.1.2 https:/ / doi.org/10.31559/glm2016.2.1.2

[10] M. Randic, On characterization of molecular branching, J. Am. Chem. Soc. 97 (1975), 6609-6615.

[11] M. Randic, Quantitative Structure-Property Relationship: boiling point and planar benzenoids, New. J. Chem. 20(1996), 1001-1009.

[12] Rachanna. Kanabur, On Certain Degree-Based Topological Indices of Armchair Polyhex Nanotubes, Journal of Mathematical Nanoscience (Accepted). https:/ / doi.org/10.21042/amns.2016.2.00029

[13] V.S Shigehalli, R.Kanabur, Degree Based Topological Indices of n- Heptane Isomers, Jamal Academic Research Journal: An Interdisciplinary Special Issue (2016), 235-238.

[14] V.S. Shigehalli, R. Kanabur, New Version of Degree-Based Topological Indices of Certain nanotube, Journal of Mathematical Nano science, 6 (1-2), (2016), 29-39.

[15] V.S. Shigehalli, R. Kanabur, Computation of New Degree-Based Topological Indices of Graphene, Journal of Mathematics, 2016. https://doi.org/10.1155/2016/4341919

[16] V.S. Shigehalli, R. Kanabur, Computing Some Degree-Based Topological Indices of Graphene, Indonesian Journal of Electrical Engineering and Informatics (IJEEI), 5 (2), (2017), 155-161. https:/ / doi.org/10.11591/ijeei.v5i2.258

[17] V.S. Shigehalli, R. Kanabur, Correlation between the Arithmetic-Geometric Indices of n-heptane Alkane Isomers, IOSR Journal of Mathematics (IOSR-JM),12(2) Ver.IV(2016), 36-39. 
[18] M. Thakur, A. Thakur, P. V. Khadikar, QSAR study of benzene sulphonamide carbonic anhydrase inhibitors: Topological approach using Balban index, Bioorg. Med. Chem. 12 (4) (2004), 789-793. https:/ /doi.org/10.1016/j.bmc.2003.10.058

[19] N.Trinajstic, Chemical Graph theory, CRC Press, Boca Raton, (1992). 